\title{
Dynamic Multi-Robot Task Allocation under Uncertainty and Temporal Constraints
}

\author{
Shushman Choudhury, Jayesh K. Gupta, Mykel J. Kochenderfer, Dorsa Sadigh, and Jeannette Bohg
}

\begin{abstract}
We consider the problem of dynamically allocating tasks to multiple agents under time window constraints and task completion uncertainty. Our objective is to minimize the number of unsuccessful tasks at the end of the operation horizon. We present a multi-robot allocation algorithm that decouples the key computational challenges of sequential decision-making under uncertainty and multi-agent coordination and addresses them in a hierarchical manner. The lower layer computes policies for individual agents using dynamic programming with tree search, and the upper layer resolves conflicts in individual plans to obtain a valid multi-agent allocation. Our algorithm, Stochastic Conflict-Based Allocation (SCoBA), is optimal in expectation and complete under some reasonable assumptions. In practice, SCoBA is computationally efficient enough to interleave planning and execution online. On the metric of successful task completion, SCoBA consistently outperforms a number of baseline methods and shows strong competitive performance against an oracle with complete lookahead. It also scales well with the number of tasks and agents. We validate our results over a wide range of simulations on two distinct domains: multi-arm conveyor belt pick-and-place and multi-drone delivery dispatch in a city.
\end{abstract}

\section{INTRODUCTION}

Efficient and high-quality task allocation is crucial for modern cooperative multi-robot applications [1]. For warehouse logistics, teams of mobile robots carry goods between assigned locations [2]. Industrial and manufacturing operations involve manipulators collaborating on assembly lines [3]. Ondemand ridesharing and delivery services dispatch agents to incoming requests [4]. Multi-robot task allocation needs to be computationally efficient and produce high-quality solutions under the challenges of real-world robotics: uncertainty of task execution success, temporal constraints such as ordering and time windows, and tasks dynamically appearing online. For instance, in one of our simulation domains, a team of robot arms pick objects that appear on a conveyor belt from an external loading process and place them in bins (Figure 1a). With time window constraints induced by workspace limits, and uncertainty due to imperfect grasping, the arms attempt to pick-and-place as many objects as possible.

Multi-robot task allocation is a difficult problem; it inherits the combinatorial optimization challenges of classical allocation as well as the uncertainty and dynamic nature of robotics. Timeextended tasks and time window constraints further require algorithms to plan over horizons rather than instantaneously, and account for spatio-temporal relationships among tasks [5]. The robotics community has worked on multi-agent task allocation with Markov Decision Processes [6] and robust task

All authors are with Stanford University, CA, USA. Please send correspondence to shushman@es.stanford.edu

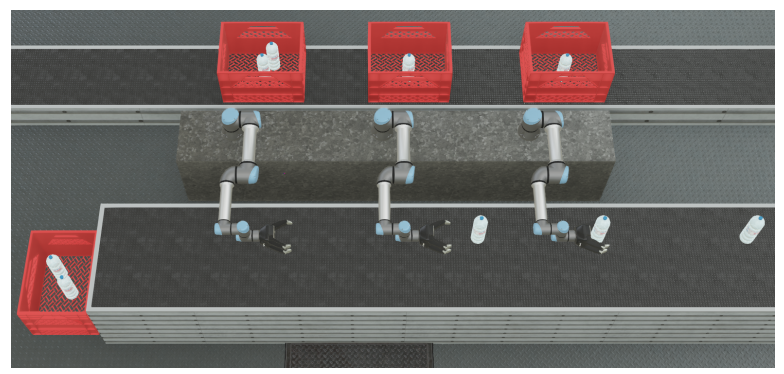

(a) Conveyor Belt Pick-and-Place

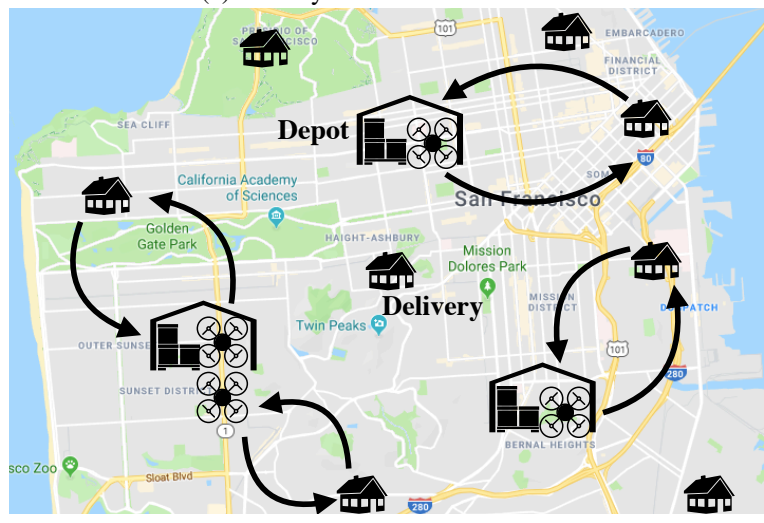

(b) Multi-Drone Delivery Dispatch

Fig. 1: The above domains motivate our multi-robot task allocation approach. We allocate robots (arms or drones) to tasks (pick-andplace or delivery) that arrive online. Task completion is subject to uncertainty (grasping or flight time) and time window constraints.

matching [7]. The classic multi-robot task allocation problem has been classified extensively [1] and extended to account for uncertainty [8], temporal and ordering constraints [5], and dynamic task arrivals [9] (some of these will be baselines for our method). The operations research community has developed methods for task execution uncertainty $[10,11]$ and online rescheduling [12]. However, their simplified agent models (such as flow shops) do not address the spatial relationships between tasks or the intersection of uncertainty and time constraints.

The algorithmic challenges for our allocation setting are sequential planning under uncertainty and coordinating multiagent decisions. Prior works typically attempt the computationally intractable joint problem and thus require simplifying approximations or heuristics for either planning or coordination. Our key idea is to decouple these challenges and address them hierarchically in an efficient two-layer approach. The lower layer plans for individual agents, using dynamic programming on a policy tree to reason about the uncertainty over task 
completion. The upper layer uses optimal conflict resolution logic from the path planning community to coordinate the multi-agent allocation decisions [13]. Our overall algorithm, Stochastic Conflict-Based Allocation (SCoBA), yields allocation policies that minimize the expected cumulative penalty for unsuccessful tasks; SCoBA can also seamlessly interleave planning and execution online for new tasks.

The following are the contributions of our work:

- We propose a general formulation for multi-robot allocation under task uncertainty and temporal constraints.

- We present a hierarchical algorithm, SCoBA, which uses multi-agent conflict resolution with single-agent policy tree search. We prove that SCoBA is both optimal in expectation and complete under mild assumptions.

- We demonstrate SCoBA's strong competitive performance against an oracle with complete lookahead, and its performance advantage over four baseline methods for successful task execution. Our results also show that SCoBA scales with increasing numbers of agents and tasks. We run simulations on two distinct robotics domains (Figure 1); a team of robot arms picking and placing objects from a conveyor belt and on-demand multi-drone delivery of packages in a city-scale area.

An extended version of this paper is available online ${ }^{1}$. The appendix contains proofs and additional evaluation details.

\section{BACKGROUND AND RELATED WORK}

We briefly discuss three background areas: i) algorithms for assignment and scheduling, ii) multi-agent decision-making, and iii) relevant work in multi-robot task allocation.

Assignment and Scheduling: A major topic in discrete optimization is assigning resources to tasks [14], for which the Hungarian algorithm is a fundamental approach [15]. In temporal tasks, we use the scheduling model, where the objective is a function of completed jobs [16]. Scheduling problems with multiple resources and tasks are computationally hard, even when deterministic [17]. In online scheduling, each task is only observed when made available [18]. Hard realtime tasks have a time window constraint for completion [19]. Approaches for scheduling under uncertainty address problems where task execution is not fully deterministic [20, 21]. These approaches are either proactive in anticipating future disruptions [22], reactive to changes [23, 24], or hybrid [25]. For scenarios with ordering constraints (such as assembly lines), additional models like job shops [26] and flow shops [27] are useful, particularly real-time flow shop scheduling [11, 28]. Iterative conflict resolution, which we adapt in our approach, was used for scheduling [29], joint task assignment and pathfinding [30], and diagnosis and repair [31]. This extensive body of work provides valuable insights but does not consider spatial relationships between tasks and their coupling with temporal uncertainty.

Multi-Agent Sequential Decision-Making: The Markov Decision Process (MDP) is a mathematical model for our setting

\footnotetext{
${ }^{1}$ https://arxiv.org/abs/2005.13109
}

of sequential decision making under uncertainty [32]. Different solution techniques exist for MDPs, depending on available information; dynamic programming [33] when the explicit transition model is known, sample-based online planning [34] when only a generative model exists, and reinforcement learning [35] when no model is available. Our problem is a multi-agent MDP (MMDP), where agents coordinate to achieve a single shared objective; planning for MMDPs is generally computationally intractable due to the exponentially large decision space [36]. Although reinforcement learning techniques are often employed to alleviate some tractability issues by learning values of different states and actions, modelfree methods like Q-Learning face exploration challenges [37, 38]. Online tree search methods can fare better by focusing on relevant states more effectively [39]. As we will demonstrate, merely framing multi-robot task allocation as an MMDP will not yield good quality solution strategies (due to its computational challenges).

Multi-Robot Task Allocation: We outline a number of domain-agnostic and domain-specific works on multi-robot task allocation. MDP solvers have been used to generate a sequential greedy strategy, but without accounting for completion uncertainty [6]. The probability of task failure has been considered by two-stage Stochastic Integer Programs and network flow algorithms, which are exhaustive combinatorial approaches unsuitable for tasks streaming in online [10, 40, 41]. A sensitivity analysis approach to optimal assignment under uncertainty provides some insights on robustness but has no notion of temporal constraints [7]. The taxonomies for multi-robot task allocation under temporal constraints help us characterize our problem's difficulty [1, 42].

Previous work on collaborative assembly lines includes hierarchical planning frameworks, constraint programming, and robust scheduling for robotic flowshops [3, 43, 44]. However, they all simplify one or more key complexities such as task completion uncertainty or multi-agent configuration models. Dynamic vehicle dispatch problems have been explored in work on vehicle routing algorithms with time windows and trip assignment algorithms for ridesharing [45, 46]. However, they make restrictive assumptions on the uncertainty and environment dynamics [5]. Driver-task assignment with uncertain durations and task windows do solve for a similar setting as ours but assume some knowledge of future requests [47].

\section{PROBLEM Formulation}

We base our formulation on previous work for multi-robot task allocation with temporal constraints [5]. There is a set of $N$ agents, denoted as $[N]$ and $K$ tasks, denoted as $[K]$; the problem horizon is $T$ time-steps. For each agent $n \in[N]$ and task $k \in[K]$, the service time window is $W_{n k}=\left(t_{n k}^{l}, t_{n k}^{u}\right)$, where $l$ and $u$ are respectively the lower and upper time limits within which $n$ can attempt $k$. There may also be an additional so-called downtime if the agent executes the task successfully, e.g., the time for a robot arm to transfer the object to the bin. We represent task duration uncertainty as

$$
\tau_{n k}(t)=\operatorname{Prob}[n \text { completes } k \text { within } t \text { time-steps }] \text {. }
$$




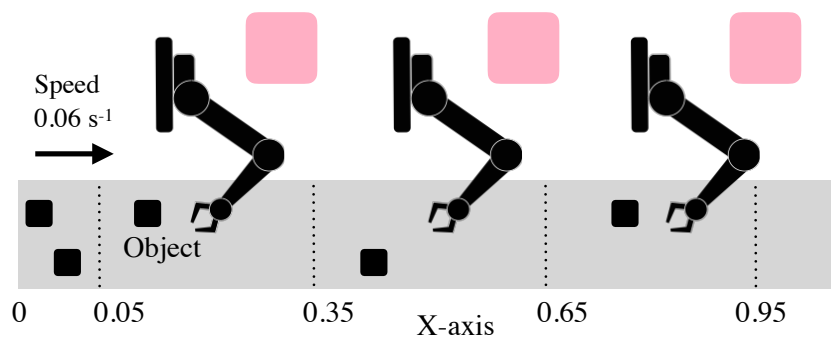

Fig. 2: The illustrated conveyor belt has $N=3$ arms and $K=5$ objects. The belt is of unit length, and each arm's workspace spans 0.3 units (dashed lines are the limits). Given the belt speed, the agent-task time window for any arm-object pair is at most $5 \mathrm{~s}$.

We assume knowledge of this cumulative distribution as part of the problem specification, typical for task scheduling under uncertainty [20]; the particular model is domain-dependent. By definition, the conjunction of $W$ and $\tau$ imposes an upper bound on task completion probability, i.e.,

$$
\text { Prob }[n \text { completes } k] \leq \tau_{n k}\left(t_{n k}^{u}-t_{n k}^{l}\right) \text {. }
$$

For all unsuccessful tasks, the system incurs a penalty of $\sum_{k} J(k)$ units. An agent can attempt only one task at a time.

We seek an allocation policy that minimizes the expected cumulative penalty due to unsuccessful tasks. An allocation policy $\pi$ is a mapping from the agents to the tasks and their respective attempt times, i.e. $\pi:[N] \rightarrow[K] \times[T]$. Since there is uncertainty about task completion, a single-shot allocation is insufficient. Of course, the attempt times for future tasks depend on when the earlier tasks are actually executed (successfully or unsuccessfully). Our optimization problem is

$$
\begin{array}{ll}
\underset{\pi \in \Pi}{\operatorname{argmin}} & \mathbb{E}\left[\sum_{k \in[K]} \mathbb{1}[k] \cdot J(k) \mid \pi\right] \\
\text { s.t. } & t \in W_{n k} \quad \forall(k, t) \in \pi(n),
\end{array}
$$

where the indicator function $\mathbb{1}[k]=1$ if the task $k$ remains incomplete at the end of the horizon, and $\Pi$ is the set of all possible allocation policies. The constraint enforces that an agent attempts a task within the valid time window. The expectation is over the task execution success distribution for the allocation policy. For the rest of the discussion, we will assume that $J(k)=1$, i.e., all tasks are equally important; this objective is the unweighted tardy jobs penalty [16].

The discrete-time rolling horizon formulation described above is fairly general and useful. We are concerned with high-level allocation rather than the underlying task execution, so we avoid the added complexity of continuous-time representations. The underlying tasks typically involve time-constrained trajectory planning, for which there are well-established models and methods [48]. Furthermore, we can interleave planning and execution suitably and recompute an allocation policy when new tasks appear online (and we do so in practice).

Motivating Examples: We describe two distinct robotics settings to instantiate our formulation. First, consider the previously introduced example of robot arms along a conveyor belt (see Figure 2). Each arm has an associated collection bin for objects picked up from the belt. The objects appear on the belt through an external process. The arms take varying amounts of time for picking, depending on the quality of the grasp strategy or gripper attributes. Arms have finite reach, and an object may not be picked up before it goes out of reach. Objects missed by all arms must be sorted by hand afterwards. The goal is to successfully pick-and-place as many objects, or equivalently, miss as few objects as possible.

Second, consider on-demand multi-drone dispatch for package delivery in a city (note the underlying similarities to the previous example). Delivery tasks arise through an external process of customer requests. Drones take varying amounts of time to travel from the product depot to the package delivery location, depending on flight conditions. Requests arrive with time windows, such that drones must wait until the window starts to deliver the product to the customer, and late deliveries are penalized. Over a certain time horizon, our objective is to minimize the number of late deliveries.

Challenges: To motivate our approach, we briefly discuss the problem complexity. By the multi-robot task allocation taxonomy of Gerkey et al. [1], the deterministic version of our problem is ST-SR-TA, i.e. a single robot (SR) executes a single task (ST) at a time, where tasks are time-extended (TA) rather than instantaneous. ST-SR-TA problems are an instance of an NP-Hard scheduling problem, specifically multi-agent scheduling with resource constraints [49]. The uncertainty of task execution success exacerbates this difficulty. Time windows make allocation harder by requiring algorithms to account for spatio-temporal task relationships [5]. Finally, new tasks streaming in require our approach to interleave planning and execution effectively, e.g., by replanning at task arrivals [9].

\section{Hierarchical Multi-Robot Task Allocation}

Our key algorithmic challenges are sequential planning under uncertainty (of task completion) and multi-agent coordination (of allocations). The joint multi-agent planning problem is computationally prohibitive for large settings [36]; most closely related previous works either use simplifying approximations for planning and optimization $[4,41]$ or simple coordination heuristics [8, 50]. In contrast, we address the challenges hierarchically in a two-layer approach called Stochastic Conflict-Based Allocation (SCoBA). At the low level, we independently determine the optimal task attempt sequence for each individual agent, ignoring other agents. At the high level, we resolve potential conflicts in assigned tasks across multiple agents to obtain a valid multi-robot allocation. In this section, we will discuss in detail the two layers and how they come together in SCoBA. We will then briefly mention how we interleave planning and execution online and how SCoBA can exploit sparse agent interactions using coordination graphs.

\section{A. Low-Level: Single Agent Policy}

We consider the perspective of an individual agent, independent of the other ones. From the definition in Section III, we have the set of current tasks, corresponding time windows, 

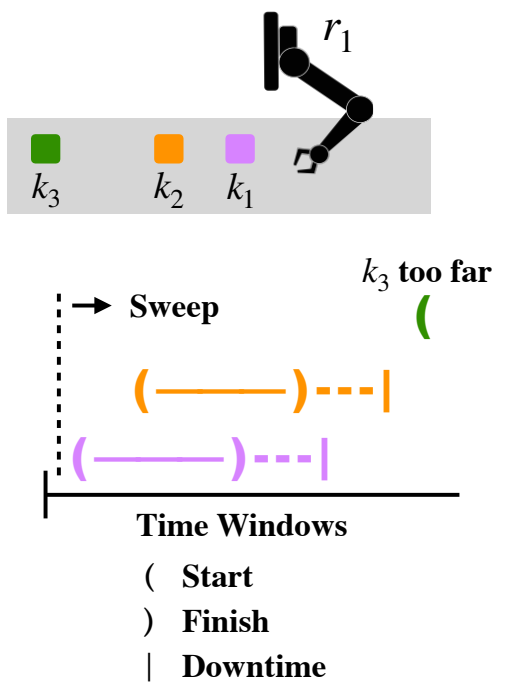

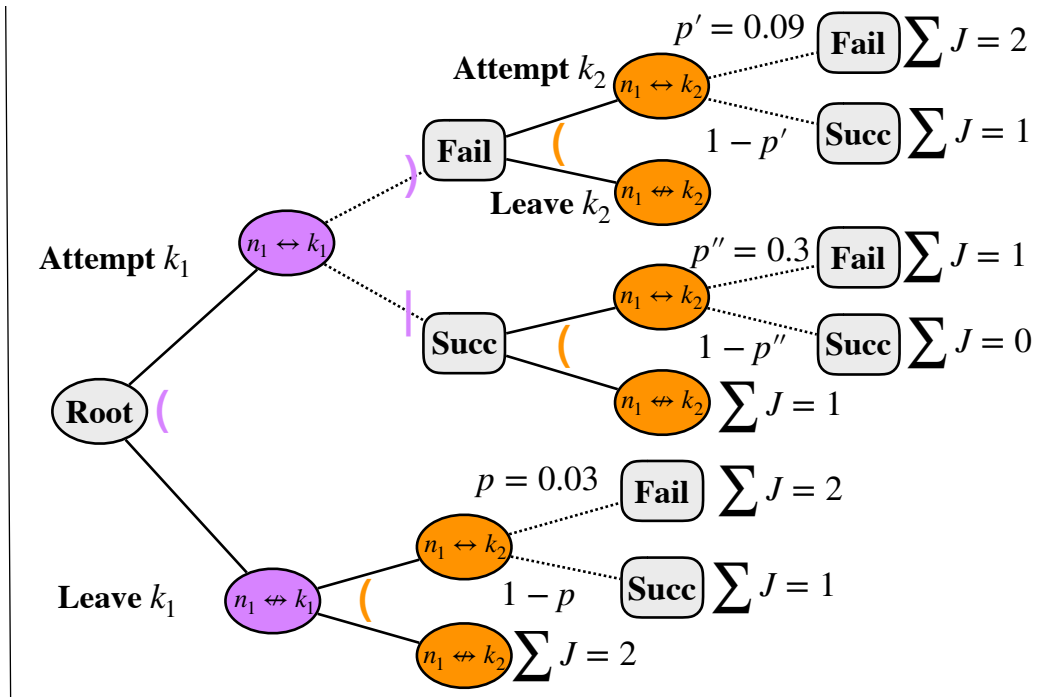

Fig. 3: The low-level routine of SCoBA generates the policy tree over valid tasks for an individual agent, specifically, by sweeping along the time axis and branching on the start or finish of a task's time window. At the start of a window, two new decision nodes (ovals) are introduced: to attempt $(\leftrightarrow)$ or to leave $(\Varangle)$ ) the task respectively. At the end of a time window and the downtime, the outcome nodes (rectangles) depict failure or success. After the tree generation, dynamic programming propagates the values from the leaves to the root. The probability values $p=0.03, p^{\prime}=0.09, p^{\prime \prime}=0.3$ are just hypothetical values that illustrate how the same attempt node $\left(n_{1} \leftrightarrow k_{2}\right)$ has three different copies, with different outcome probabilities (depending on the branch of the tree).

and task completion uncertainty distribution, and we want a task attempt policy tree for the agent. Since task execution is stochastic, the first possible attempt time of a task depends on the tasks attempted before it. We make a simplifying approximation - the agent attempts a task as soon as possible and observes the outcome at the end of the window. This approximation collapses the temporal dimension by treating tasks as discrete events rather than extended ones.

We illustrate the policy tree search process for a single robot $n_{1}$ and three tasks (objects) $k_{1}, k_{2}, k_{3}$ in Figure 3 . First, we sort tasks in increasing order of the start of their time window. Then, we sweep along the time axis and update the tree at every event point, i.e., the start or finish of the window (and the end of the downtime if the task were to be successful). The updates to the policy tree depends on the event point (start/finish/downtime). For the start of a time window, we introduce two new decision nodes (ovals) to attempt $(\leftrightarrow)$ or leave ( $\measuredangle)$ the task respectively. At the end of a time window and the downtime, we introduce outcome nodes (rectangles) respectively for failure or success, where the outcome probability $p$ depends on the minimum feasible start time for the attempt, which in turn depends on the specific branch of the tree. For instance, notice in Figure 3 the three copies of the decision node $\left(n_{1} \leftrightarrow k_{2}\right)$, with different probabilities, depending on whether it was attempted after the failure, success or non-attempt of task $k_{1}$.

The leaves of the binary policy tree contain the cumulative penalty along their branches, e.g., a penalty of 1 for each unsuccessful task. We then use dynamic programming to propagate values upwards from the leaves to the root. For a pair of outcome node siblings, we set the parent's value (denoted as $V$ ) to the expected value of its children,

$$
V(\text { parent }):=p \cdot V(\text { Fail })+(1-p) \cdot V(\text { Succ }) \text {. }
$$

For a pair of decision node siblings, the parent's value is the minimum of the children's, i.e.,

$$
V(\text { parent }):=\min \{V(\text { child } 1), V(\text { child } 2)\}
$$

in the running example in Figure 3, we have $V$ (root) $=$ $\min \left\{V\left(n_{1} \leftrightarrow k_{1}\right), V\left(n_{1} \nLeftarrow \neg k_{1}\right)\right\}$. The resulting tree encodes the policy that minimizes the agent's expected penalty for all tasks up to the planning horizon, and $V$ (root) is the value of this expected penalty. We obtain the next task assigned to the agent by following child nodes of minimum value until the first attempt node (e.g., $n_{1} \leftrightarrow k_{1}$ ).

\section{B. High-Level: Multi-Agent Coordination}

The policy tree determines the approximately optimal task attempt sequence for an individual agent (approximate due to the temporal simplification mentioned earlier). The tree searches are independent of each other, so two agents may have conflicting allocations. Since our objective function depends on all agents, breaking ties naïvely could yield arbitrarily poor global allocations. Multi-agent pathfinding algorithms face a similar challenge and have to resolve inter-agent conflicts between shortest paths [51]. Conflict-Based Search is an effective strategy for this problem [13]; by decoupling singleagent path planning and inter-path conflict resolution, it is efficient in practice without losing optimality.

We leverage the idea of inter-agent conflict resolution from Conflict-Based Search. The high level of our algorithm, SCoBA, searches a binary constraint tree (Figure 4) generated from conflicts between solutions for individual agents obtained from the low level, i.e., the policy tree search. Two agents $n_{1}$ and $n_{2}$ are in conflict if they are allocated the same task $k$ in overlapping time windows, i.e., if $\left(k, t_{1}\right) \in \pi\left(n_{1}\right),\left(k, t_{2}\right) \in$ $\pi\left(n_{2}\right)$ and either $t_{2} \in W_{n_{1}, k}$ or $t_{1} \in W_{n_{2}, k}$. A constraint 


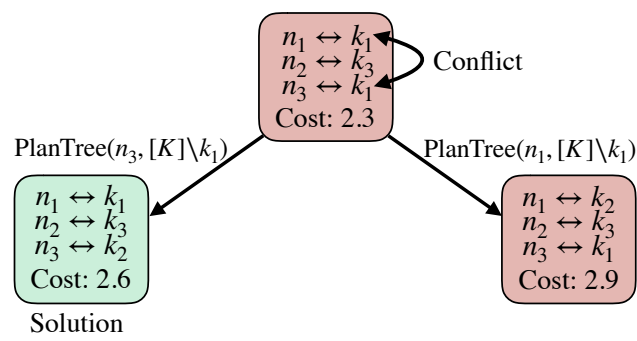

Fig. 4: A constraint tree node with a conflict in the allocation generates two children with corresponding constraints on the conflicting agents ( $n_{1}$ and $\left.n_{3}\right)$ and task $\left(k_{1}\right)$. Best-first search on the constraint tree returns the first high-level node with a conflict-free allocation.

for an agent is a task excluded from consideration by the tree search for that agent. Each node in the constraint tree maintains (i) a set of constraints, i.e., tasks to ignore, for each agent, (ii) a multi-agent allocation that respects all constraints, and (iii) the cost of the allocation. For SCoBA, the cost of the allocation is the sum of expected penalties for each agent, where the expected penalty for each agent is the value of the root node of its policy tree. The allocation cost is used as the criteria for best-first search on the constraint tree; this best first search continues until it finds a conflict-free allocation.

\section{Stochastic Conflict-Based Allocation (SCoBA)}

Algorithm 1 describes SCoBA, using the tree search of Section IV-A as the PLANTREE subroutine. Its structure is similar to a presentation of Conflict-Based Search by Felner et al. [51]. The constraint tree is initialized with the root node, which has an empty constraint set and the allocation from running PLANTREE for each individual agent (lines 2-6). When a high-level node is expanded, the corresponding allocation is checked for validity (line 9). If there is no conflict, this allocation is returned as the solution. Otherwise, for every conflict between two or more agents, new child nodes are added, where constraints are imposed on the agents involved (line 14). A child constraint tree node inherits its parent's constraints and adds one more constraint for a particular agent.

Consider the simple illustrative example in Figure 4. The root node has agents $n_{1}$ and $n_{3}$ both assigned to task $k_{1}$. This conflict yields two constraints, one inherited by each of the two child nodes. The first constraint excludes $k_{1}$ from the recomputed policy tree search for $n_{1}$. The second constraint does the same for $n_{3}$. For each new (non-root) node, the low level tree search is only re-run on the agent for which the constraint is added (line 18). Both of the resulting child nodes are conflict-free, but the left one, with a lower allocation cost of 2.6 , is returned as the solution.

Our problem setting is both online and stochastic. However, under some simplifying assumptions, we can establish optimality and completeness properties for SCoBA.

Proposition 1. If (i) no new tasks are added online, (ii) the tree search is executed to the full horizon, and (iii) task completion

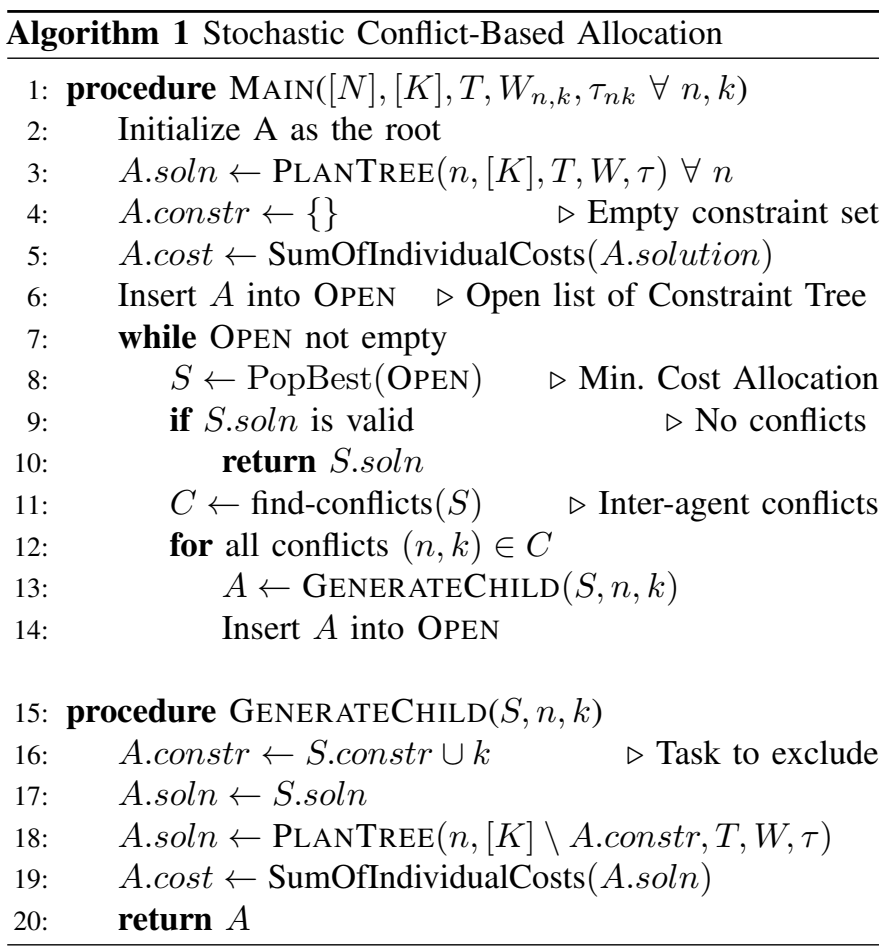

is determined at the end of the time window, then SCoBA is optimal in expectation, i.e. SCoBA minimizes in expectation the number of incomplete tasks at the end of the time horizon.

Proposition 2. Under the assumptions of Proposition 1, SCoBA is complete. If a valid allocation exists, SCoBA returns it.

We provide detailed proofs in the appendix. We derive them from the corresponding optimality and completeness proofs of the Conflict-Based Search algorithm for multi-agent pathfinding [13]. For optimality, we use existing results in sequential decision-making to show that SCoBA's low-level routine, i.e., policy tree generation, is optimal in expectation for an individual agent [32]. We then prove that SCoBA's highlevel multi-agent coordination satisfies the sufficient condition to inherit the multi-agent optimality of Conflict-Based Search. For completeness, we show how the high-level constraint tree of SCoBA, as in Conflict-Based Search, has a finite number of nodes. Therefore, systematic best-first search on it will find a valid solution if one exists.

Interleaving Planning and Execution: To account for new tasks beyond the horizon, we interleave planning and execution online. SCoBA's elegant representation makes interleaving straightforward at both levels. For the single agent policy tree search, we truncate the search horizon based on computation requirements. In our implementation, we run the sweep until the first task whose time window begins after the downtime of all tasks before it ( $k_{3}$ in Figure 3$)$. For the multi-agent coordination, we set a threshold on the number of high-level conflicts, once again based on real-time computation constraints. If the threshold is exceeded, we return the current high-level solution. For agents allocated to the same task, we break ties arbitrarily and keep the unassigned agents for allocation to new tasks at the next timestep. 
Coordination Graphs: SCoBA works with any arbitrary configuration of agents and inter-agent constraints. We also use coordination graphs (CGs) from multi-agent decision-making for greater efficiency [52]. In CGs, each node represents an agent, and each edge encodes a (potentially directed) dependency between agents, such that only connected agents need to coordinate actions (or allocations in our case). The choice of coordination graph for a problem is domain-dependent and often quite natural. For instance, in the conveyor belt example, the arms are ordered along the belt and their workspaces are mutually exclusive, therefore the coordination graph is a directed chain from the first arm to the last.

The CG structure impacts the high-level multi-agent coordination stage of SCoBA. The absence of an edge between two agents implies that their sets of possible tasks are disjoint, i.e., they cannot have conflicting allocations. Therefore, in practice, SCoBA need not consider dependencies between all the agents. If the CG is directed (as in the conveyor belt), we run the tree search for agents along a topological ordering of the CG. For any agent, we exclude the tasks already assigned to its predecessors. By construction, we will obtain a conflict-free allocation at the end (without any child nodes being generated in the high-level constraint tree). If the CG is undirected (as in our multi-drone delivery domain), such a topological ordering is not feasible, and conflicts may be unavoidable. However, if the CG has multiple connected components, then nodes (agents) in different components cannot conflict with each other, so we can run SCoBA on each component in parallel.

\section{Evaluation}

The primary metric for evaluating SCoBA is the accumulated penalty for unsuccessful tasks. We will also evaluate its scalability to tasks and agents via computation time. We first outline the range of methods we use to baseline SCoBA. We then present and discuss the results for both performance metrics on simulations for each of our two distinct roboticsinspired domains: conveyor belt pick-and-place and on-demand multi-drone delivery dispatch. We use Julia [53] on a $16 \mathrm{GiB}$ RAM machine and a 6 -core $3.7 \mathrm{GHz}$ CPU for all simulations ${ }^{2}$.

\section{A. Baselines for Unsuccessful Task Penalty}

We use multiple complementary methods to baseline SCoBA on our primary metric of unsuccessful task penalty:

1) EDD: The Earliest Due Date heuristic assigns each agent to the task with the nearest time window deadline and is a common heuristic for scheduling [16].

2) Hungarian: An unbalanced Hungarian algorithm, where the edge weight for an agent-task pair is the probability of successful task completion [15]. This method is a special case of a general purpose network-flow approach, where only one task is assigned at a time [41].

3) Q-Learning: We frame the multi-robot task allocation problem as a discrete-time Markov Decision Process and pre-compute a policy with Q-Learning.

\footnotetext{
${ }^{2}$ The code is available at https://github.com/sisl/SCoBA.jl
}

4) MCTS: A recent Monte-Carlo Tree Search approach specifically for multi-robot task allocation [50]. The tree search is conceptually similar to ours (albeit with Monte Carlo sampling of outcomes) but it uses arbitrary priority orderings among agents to coordinate decisions and control the tree branching factor.

For the baselines, we cover a range of approaches for multirobot allocation from scheduling to sequential decision-making under uncertainty. Both EDD and Hungarian are reactive, i.e., do not plan sequentially. The latter optimizes for multiple agents, unlike the former. Both Q-Learning and MCTS plan sequentially by framing an MDP, but the former is model-free and offline while the latter is model-based and online.

\section{B. Conveyor Belt: Experiments and Results}

Three robot arms are arranged along a moving conveyor belt, picking objects from the belt and placing them in collection bins (Figure 1a). We design an abstracted simulation of the scenario (Figure 2), scaled along an $\mathrm{X}$-axis of unit length. The arms have mutually exclusive adjacent workspaces of 0.3 units each, from $x=0.05$ to $x=0.95$. New tasks arrive as new objects appear at the head of the belt. Three scenario parameters instantiate the problem and affect the difficulty:

- Grasp Success Probability: We model uncertainty over task completion due to imperfect grasping with a Bernoulli process where $p_{i}$ is the probability of a successful pick by the $i$ th arm. Accordingly, the cumulative distribution from Equation (1) is

$$
\tau_{n k}(t)=1-\left(1-p_{i}\right)^{t}, \quad t \in \mathbb{N} .
$$

We expect performance to improve as $p_{i}$ increases.

- Belt Speed: The speed of the belt determines the effective time window for each arm-object pair, e.g., $5 \mathrm{~s}$ in Figure 2. If task execution is successful, each arm has a downtime of $\Delta t=2 \mathrm{~s}$ to deposit the object in the bin. We expect the performance to degrade as speed increases.

- New Object Probability: We briefly describe how new objects arrive (more details in the appendix). We reflect the setup in space (about Y-axis) and time, with virtual arms operating in reverse, transferring objects from virtual bins to a virtual belt. Upon crossing the Y-axis, the virtual belt becomes the true belt and virtual objects appear as real ones. The new object probability parameter is the per-timestep Bernoulli probability with which a virtual arm drops its object onto the virtual belt (all virtual arms have the same such probability). The drop location is sampled uniformly within the virtual arm's workspace. As soon as the virtual arm drops an object, it moves to the virtual bin to collect the next one. We expect performance to degrade as new object probability increases.

Competitive Performance against Oracle: A standard metric for online algorithms is the competitive performance against an oracle with complete lookahead. The task generation process allows an ablation study for the effect of lookahead alone (decoupled from the effect of uncertainty). If grasping is perfect, 

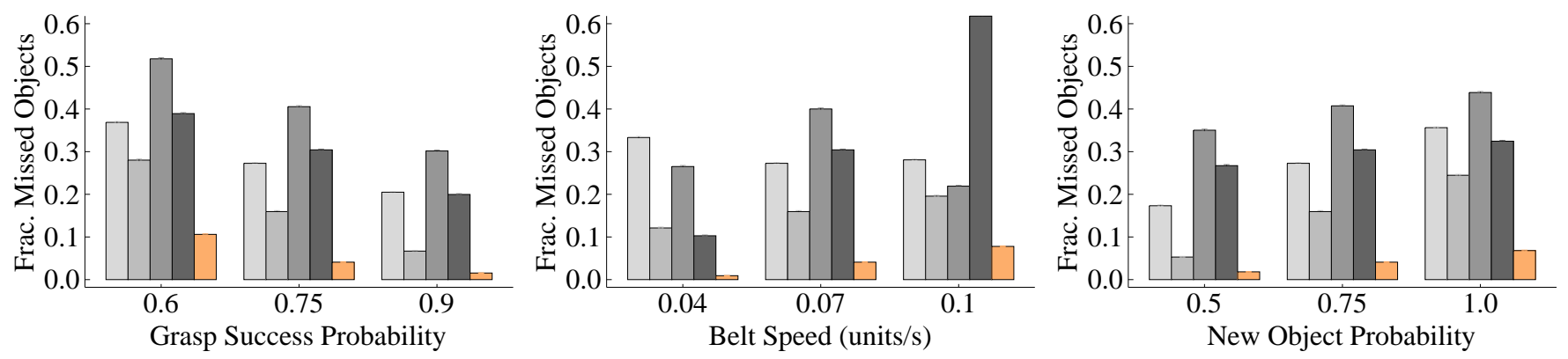

Fig. 5: Legend: $\square$ EDD $\square$ Hungarian $\square$ MCTS $\square$ Q-Learning $\square$ SCoBA. On the metric of the fraction of unsuccessful tasks, i.e. objects missed, SCoBA consistently outperforms all other baselines. All results are averaged over 100 trials, with $T=500$ time-steps per trial.

TABLE I: Average proportions of objects lost per trial by SCoBA when grasping is perfect. The two varied parameters affect SCoBA's lookahead. The negligible values demonstrate the strong competitive performance of SCoBA relative to the oracle.

\begin{tabular}{lrrr}
\hline Belt Speed & \multicolumn{3}{c}{ New Object Probability } \\
\cline { 2 - 4 } (units/s) & 0.5 & 0.75 & 1.0 \\
\hline 0.04 & 0.0 & 0.0 & 0.0 \\
0.07 & $2.7 \times 10^{-5}$ & $7.9 \times 10^{-5}$ & $1.3 \times 10^{-4}$ \\
0.1 & $1.7 \times 10^{-4}$ & $3.7 \times 10^{-4}$ & $5.2 \times 10^{-4}$ \\
\hline
\end{tabular}

i.e., $p_{i}=1$ for all arms, there exists an oracle strategy that can complete all tasks successfully. The oracle is that which mirrors the generation sequence itself in space and time. We compare SCoBA (without full lookahead) to this oracle by evaluating the proportion of tasks it fails to complete with this generation process. The smaller this number, the better is SCoBA's competitive performance. We set $p_{i}=1$ for all arms and jointly vary the other two parameters, belt speed and new object probability. We choose a maximum belt speed of 0.1 units/s so that each object spends at least $2 \mathrm{~s}$ in an arm's workspace. For each trial in a setting, we simulate $T=500$ time-steps (seconds) and evaluate the proportion of objects missed by SCoBA relative to the total number of objects. We compute the average of this proportion-per-trial over 100 trials (standard error negligible) in Table I. The low magnitudes demonstrate SCoBA's robustness to insufficient lookahead. With increasing value of either parameter, performance degrades. Unsuccessful Task Penalty: We vary all three scenario parameters independently and compare the fraction of missed objects for SCoBA versus the other baselines. Figure 5 demonstrates the results. For each subplot, only one parameter varies (the $\mathrm{x}$-label), while the other two stay at their default values - grasp probability $p_{i}=0.75$ for all arms, 0.07 units/s for belt speed, and 0.75 for new object probability. We average all numbers over 100 trials (with standard error bars).

SCoBA considerably outperforms the other baselines across all settings. Furthermore, its performance degrades or improves as expected relative to the change in each problem parameter (e.g., more objects missed with increasing new object probability). Among the baselines, the reactive Hungarian method has the best performance, likely because sequential deliberation is not as crucial with non-overlapping workspaces and small downtime (unlike in the next domain). For Q-Learning and
TABLE II: The low computation time values demonstrate that the tree search for an individual arm is quite scalable with respect to the number of objects in the arm's workspace.

\begin{tabular}{lrr}
\hline Objects & Tree Size & Comp. Time \\
\hline 40 & 640.9 & $9 \times 10^{-4} \mathrm{~s}$ \\
80 & 2215.3 & $0.004 \mathrm{~s}$ \\
120 & 5102.3 & $0.013 \mathrm{~s}$ \\
160 & 8791.7 & $0.029 \mathrm{~s}$ \\
200 & 13028.4 & $0.051 \mathrm{~s}$ \\
\hline
\end{tabular}

MCTS, the performance depends on how finely the conveyor belt is discretized. In Q-Learning, the entire state space also needs to be explicitly enumerated. We used a discretization of 0.05 units for Q-Learning and 0.02 units for MCTS; too much finer would result in a prohibitively large state space due to the curse of dimensionality [32].

Scalability: In this domain, the Coordination Graph is a directed chain (see Section IV), so the computational bottleneck for SCoBA is the policy tree search (multi-agent coordination is trivial). In Table II we report average tree search computation times for a single arm with an increasing number of objects (scattered throughout the arm workspace). Empirically, we observe that the number of tree nodes is roughly quadratic, and the computation time roughly cubic in the number of objects, and the wall clock times are quite reasonable. The appendix has further timing results for the applicable baselines.

\section{Drone Delivery: Experiments and Results}

In the second domain, we dispatch drones to deliver packages around a city-scale area, subject to delivery time window constraints. Our setup is based on our recent work for multidrone delivery over ground transit [54]. We use the locationto-location travel time estimates from its North San Francisco scenario, which simulates deliveries over an area of $150 \mathrm{~km}^{2}$ (see Figure 1b). We handpick locations for up to 5 depots scattered around the city to ensure good coverage. Drones have a maximum flight range of $10 \mathrm{~km}$, which restricts the set of possible package deliveries for each drone. Two scenario parameters affect the performance here:

- Drones and Depots: The number of depots and the ratio of drones to depots both impact the ability of the system to dispatch agents to a given delivery location in time. We distribute drones equally across depots. With better coverage, we expect performance to improve. 

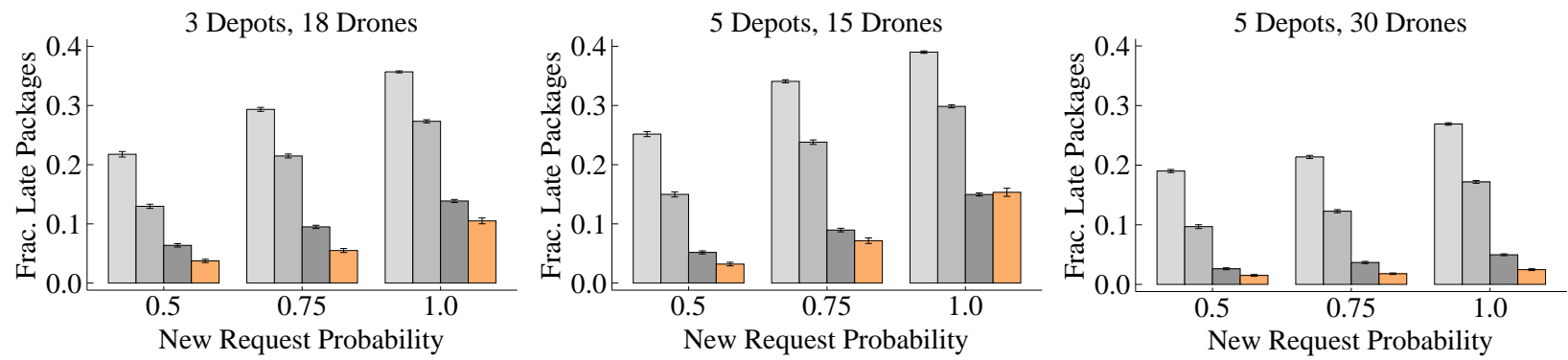

Fig. 6: Legend: $\square$ EDD $\square$ Hungarian $\square$ MCTS $\square$ SCoBA. For the drone delivery domain, on the primary metric of the fraction of late package deliveries, SCoBA outperforms the baselines on all but one setting. Results are averaged over 100 trials each of $T=100$ time-steps.

- New Request Probability: A new delivery request arrives per minute with some probability. Each delivery location is sampled uniformly within a bounding box. We start with a number of packages roughly 1.5 times the number of drones in the scenario. With higher probability, we expect performance to degrade. Each request has a window duration sampled uniformly between 15 and 30 minutes.

Our reference framework gives us deterministic drone traveltime estimates between a depot $d$ and package location $p$, say $T T(d, p)$. We model travel time uncertainty with a finitesupport Epanechnikov distribution around $T T(d, p)$, i.e.,

$$
\tau_{n, k}(t) \sim \operatorname{Epan}(\mu=T T(d, p), r=T T(d, p) / 3.0) .
$$

The true travel time is drawn from Equation (7). This choice of a synthetic distribution is arbitrary but reasonable because a high mean travel time is likely to have higher variance, due to more opportunities for delays or speedups.

1) Unsuccessful Task Penalty: We vary the scenario parameters and compare the fraction of late package deliveries for SCoBA versus the other baselines in Figure 6. We choose three sets of depot-and-drone numbers with complementary coverage properties, e.g., $(3,18)$ has fewer depots and a higher dronedepot ratio while $(5,15)$ has more depots but a smaller ratio. We vary the new request probability, simulate $T=100$ timesteps (minutes) per trial, and average results over 100 trials. We omit Q-Learning because the enumeration of the multi-agent MDP state space is unacceptably large for any useful time-axis discretization. SCoBA is generally the best across all settings except in one (5 depots, 15 drones, probability 1.0) where MCTS is slightly better. The vast improvement in relative performance of the MCTS baseline [50] is not surprising. It is tailored for vehicle dispatch problems, with search heuristics that exploit the domain structure, e.g., agents have longer downtime to return to their depots; the per-agent action space is a subset of valid tasks rather than a discretization of a conveyor belt into slots. For the coverage parameter, having more drones per depot appears to be more influential than having more depots, e.g., the errors for $(5,15)$ are higher than the corresponding ones for $(3,18)$.

2) Scalability: In this domain, high-level conflicts may occur, and SCoBA will invoke its multi-agent coordination layer (over and above policy tree search) while computing a valid multi-agent allocation. Therefore, in Table III we report the mean and standard error for computation times
TABLE III: The mean and standard error (over 50 trials in each setting) for SCoBA computation times on the multi-drone delivery domain. All times are in seconds.

\begin{tabular}{lrrr}
\hline & \multicolumn{3}{c}{ Number of Requests } \\
\cline { 2 - 4 } (Depots,Drones) & 20 & 50 & 100 \\
\hline$(3,18)$ & $(0.02,0.003)$ & $(1.48,0.16)$ & $(2.55,0.23)$ \\
$(5,15)$ & $(0.06,0.008)$ & $(2.13,0.2)$ & $(5.42,0.5)$ \\
$(5,30)$ & $(0.17,0.003)$ & $(1.76,0.12)$ & $(7.08,0.47)$ \\
\hline
\end{tabular}

for the full SCoBA algorithm (over 50 different trials for each setting). We vary the number of drones and depots and the number of currently available tasks, i.e., the current package delivery requests. The absolute wall clock values are quite reasonable given that the time-scale of operation of the system in the real world is minutes and hours. Some scenarios have disproportionately high mean and variance because of more high-level conflicts, a known behavioral property of ConflictBased Search algorithms [13]. The appendix has timing results for other baselines.

\section{CONCLUSION}

We presented SCoBA, a hierarchical approach for dynamic multi-robot task allocation under uncertainty and temporal constraints. In theory, SCoBA is optimal in expectation and complete under mild technical assumptions. In practice, over two distinct and realistic domains, it has strong competitive performance against an oracle, consistently outperforms a number of baselines, and is scalable in terms of computation time to both agents and tasks.

Limitations and Future Work: We assume a known uncertainty model, which is typical for multi-robot task allocation research. However, since SCoBA is based on policy tree search, we could use it in-the-loop with model-based RL in case the uncertainty model needs to be estimated online. SCoBA's computation time is sensitive to the number of high-level conflicts; future work could incorporate efficiency improvements to Conflict-Based Search such as bounded suboptimal variants [55] and improved conflict resolution [56]. Finally, we focus on high-level allocation here, but we could integrate $\mathrm{SCOBA}$ in a full pipeline for robotics applications.

\section{ACKNOWLEDGMENTS}

This work was supported by the Ford Motor Company, NSF grant number 1941722 and NSF grant number 1849952. 


\section{REFERENCES}

[1] B. P. Gerkey and M. J. Mataric, "A formal analysis and taxonomy of task allocation in multi-robot systems," International Journal of Robotics Research, vol. 23, no. 9, pp. 939-954, 2004.

[2] Z. Yan, N. Jouandeau, and A. A. Chérif, "Multi-robot heuristic goods transportation," in IEEE International Conference on Intelligent Systems, 2012, pp. 409-414.

[3] L. Johannsmeier and S. Haddadin, "A hierarchical human-robot interaction-planning framework for task allocation in collaborative industrial assembly processes," IEEE Robotics and Automation Letters, vol. 2, no. 1, pp. 41-48, 2016.

[4] M. Hyland and H. S. Mahmassani, "Dynamic autonomous vehicle fleet operations: Optimization-based strategies to assign AVs to immediate traveler demand requests," Transportation Research Part C: Emerging Technologies, vol. 92, pp. 278-297, 2018.

[5] M. L. Gini, "Multi-robot allocation of tasks with temporal and ordering constraints," in AAAI Conference on Artificial Intelligence (AAAI), 2017, pp. 4863-4869.

[6] T. Campbell, L. Johnson, and J. P. How, "Multiagent allocation of Markov decision process tasks," in American Control Conference (ACC), IEEE, 2013, pp. 2356-2361.

[7] L. Liu and D. A. Shell, "Assessing optimal assignment under uncertainty: An interval-based algorithm," The International Journal of Robotics Research, vol. 30, no. 7, pp. 936-953, 2011.

[8] M. J. Mataric, G. S. Sukhatme, and E. H. Østergaard, "Multi-robot task allocation in uncertain environments," Autonomous Robots, vol. 14, no. 2-3, pp. 255-263, 2003.

[9] J.-F. Cordeau and G. Laporte, "The dial-a-ride problem: Models and algorithms," Annals of Operations Research, vol. 153, no. 1, pp. 29-46, 2007.

[10] S. Timotheou, "Asset-task assignment algorithms in the presence of execution uncertainty," The Computer Journal, vol. 54, no. 9, pp. 1514-1525, 2010.

[11] D. Rahmani and M. Heydari, "Robust and stable flow shop scheduling with unexpected arrivals of new jobs and uncertain processing times," Journal of Manufacturing Systems, vol. 33, no. 1, pp. 84-92, 2014.

[12] R. O’Donovan, R. Uzsoy, and K. N. McKay, "Predictable scheduling of a single machine with breakdowns and sensitive jobs," International Journal of Production Research, vol. 37, no. 18, pp. 4217-4233, 1999.

[13] G. Sharon, R. Stern, A. Felner, and N. Sturtevant, "Conflict-based search for optimal multi-agent path finding," in AAAI Conference on Artificial Intelligence (AAAI), 2012.

[14] R. E. Burkard, M. Dell'Amico, and S. Martello, Assignment Problems. SIAM, 2009, ISBN: 978-0-89871-663-4.

[15] J. Munkres, "Algorithms for the assignment and transportation problems," Journal of the society for industrial and applied mathematics, vol. 5, no. 1, pp. 32-38, 1957.

[16] M. Pinedo, Scheduling. Springer, 2012, vol. 29.
[17] J. K. Lenstra, A. R. Kan, and P. Brucker, "Complexity of machine scheduling problems," in Annals of Discrete Mathematics, vol. 1, Elsevier, 1977, pp. 343-362.

[18] S. Albers, "Better bounds for online scheduling," SIAM Journal on Computing, vol. 29, no. 2, pp. 459-473, 1999.

[19] M. L. Dertouzos and A. K. Mok, "Multiprocessor online scheduling of hard-real-time tasks," IEEE Transactions on Software Engineering, vol. 15, no. 12, pp. 1497-1506, 1989.

[20] T. Chaari, S. Chaabane, N. Aissani, and D. Trentesaux, "Scheduling under uncertainty: Survey and research directions," in International Conference on Advanced Logistics and Transport, ICALT, 2014, pp. 229-234.

[21] D. Sadigh and A. Kapoor, "Safe control under uncertainty with probabilistic signal temporal logic," in Proceedings of Robotics: Science and Systems (RSS), Jun. 2016.

[22] X. Lin, S. L. Janak, and C. A. Floudas, "A new robust optimization approach for scheduling under uncertainty: I. bounded uncertainty," Computers \& Chemical Engineering, vol. 28, no. 6-7, pp. 1069-1085, 2004.

[23] E. Szelke and R. M. Kerr, "Knowledge-based reactive scheduling," Production Planning \& Control, vol. 5, no. 2, pp. 124-145, 1994.

[24] V. Raman, A. Donzé, D. Sadigh, R. M. Murray, and S. A. Seshia, "Reactive synthesis from signal temporal logic specifications," in Proceedings of the 18th international conference on hybrid systems: Computation and control, 2015, pp. 239-248.

[25] L. K. Church and R. Uzsoy, "Analysis of periodic and event-driven rescheduling policies in dynamic shops," International Journal of Computer Integrated Manufacturing, vol. 5, no. 3, pp. 153-163, 1992.

[26] N. Al-Hinai and T. Y. ElMekkawy, "Robust and stable flexible job shop scheduling with random machine breakdowns using a hybrid genetic algorithm," International Journal of Production Economics, vol. 132, no. 2, pp. 279-291, 2011.

[27] E. González-Neira, J. Montoya-Torres, and D. Barrera, "Flow-shop scheduling problem under uncertainties: Review and trends," International Journal of Industrial Engineering Computations, vol. 8, no. 4, pp. 399-426, 2017.

[28] J. M. Framinan, V. Fernandez-Viagas, and P. PerezGonzalez, "Using real-time information to reschedule jobs in a flowshop with variable processing times," Computers \& Industrial Engineering, vol. 129, pp. 113-125, 2019.

[29] S. Gay, R. Hartert, C. Lecoutre, and P. Schaus, "Conflict ordering search for scheduling problems," in International Conference on Principles and Practice of Constraint Programming, Springer, 2015, pp. 140-148.

[30] H. Ma and S. Koenig, "Optimal target assignment and path finding for teams of agents," in International 
Conference on Autonomous Agents and Multiagent Systems (AAMAS), 2016, pp. 1144-1152.

[31] S. Ghosh, D. Sadigh, P. Nuzzo, V. Raman, A. Donzé, A. L. Sangiovanni-Vincentelli, S. S. Sastry, and S. A. Seshia, "Diagnosis and repair for synthesis from signal temporal logic specifications," in Proceedings of the 19th International Conference on Hybrid Systems: Computation and Control, 2016, pp. 31-40.

[32] M. J. Kochenderfer, Decision Making under Uncertainty: Theory and Application. MIT Press, 2015.

[33] D. P. Bertsekas, Dynamic Programming and Optimal Control. Athena Scientific, 2005.

[34] L. Péret and F. Garcia, "Online resolution techniques," Markov Decision Processes in Artificial Intelligence, pp. 153-184, 2013.

[35] R. S. Sutton and A. G. Barto, Reinforcement Learning: An Introduction. MIT press, 2018.

[36] C. Boutilier, "Planning, learning and coordination in multiagent decision processes," in Proceedings of the 6th conference on Theoretical aspects of rationality and knowledge, Morgan Kaufmann Publishers Inc., 1996, pp. 195-210.

[37] M. L. Littman, "Markov games as a framework for multiagent reinforcement learning," in Machine Learning, Elsevier, 1994, pp. 157-163.

[38] M. Lanctot, V. Zambaldi, A. Gruslys, A. Lazaridou, K. Tuyls, J. Pérolat, D. Silver, and T. Graepel, "A unified game-theoretic approach to multiagent reinforcement learning," in Advances in Neural Information Processing Systems, 2017, pp. 4190-4203.

[39] T. Vodopivec, S. Samothrakis, and B. Ster, "On Monte Carlo tree search and reinforcement learning," Journal of Artificial Intelligence Research, vol. 60, pp. 881-936, 2017.

[40] S. Ahmed and R. Garcia, "Dynamic capacity acquisition and assignment under uncertainty," Annals of Operations Research, vol. 124, no. 1-4, pp. 267-283, 2003.

[41] S. Timotheou, "Network flow approaches for an assettask assignment problem with execution uncertainty," in Computer and Information Sciences, Springer, 2011, pp. 33-38.

[42] E. Nunes, M. D. Manner, H. Mitiche, and M. L. Gini, "A taxonomy for task allocation problems with temporal and ordering constraints," Robotics and Autonomous Systems, vol. 90, pp. 55-70, 2017.

[43] J. K. Behrens, R. Lange, and M. Mansouri, "A constraint programming approach to simultaneous task allocation and motion scheduling for industrial dual-arm manipulation tasks," in IEEE International Conference on Robotics and Automation (ICRA), IEEE, 2019, pp. 87058711.

[44] A. Che, V. Kats, and E. Levner, "An efficient bicriteria algorithm for stable robotic flow shop scheduling,"
European Journal of Operational Research, vol. 260, no. 3, pp. 964-971, 2017.

[45] H. C. Lau, M. Sim, and K. M. Teo, "Vehicle routing problem with time windows and a limited number of vehicles," European Journal of Operational Research, vol. 148, no. 3, pp. 559-569, 2003.

[46] J. Alonso-Mora, S. Samaranayake, A. Wallar, E. Frazzoli, and D. Rus, "On-demand high-capacity ride-sharing via dynamic trip-vehicle assignment," Proceedings of the National Academy of Sciences, vol. 114, no. 3, pp. 462467, 2017.

[47] R. K. Cheung, D. D. Hang, and N. Shi, "A labeling method for dynamic driver-task assignment with uncertain task durations," Operations Research Letters, vol. 33, no. 4, pp. 411-420, 2005.

[48] J.-P. Laumond et al., Robot Motion Planning and Control. Springer, 1998, vol. 229.

[49] M. R. Garey and D. S. Johnson, "Complexity results for multiprocessor scheduling under resource constraints," SIAM Journal on Computing, vol. 4, no. 4, pp. 397-411, 1975.

[50] B. Kartal, E. Nunes, J. Godoy, and M. L. Gini, "Monte Carlo tree search for multi-robot task allocation," in AAAI Conference on Artificial Intelligence (AAAI), 2016, pp. 4222-4223.

[51] A. Felner, R. Stern, S. E. Shimony, E. Boyarski, M. Goldenberg, G. Sharon, N. Sturtevant, G. Wagner, and P. Surynek, "Search-based optimal solvers for the multiagent pathfinding problem: Summary and challenges,' in Symposium on Combinatorial Search, 2017.

[52] J. R. Kok, M. T. Spaan, and N. Vlassis, "Multirobot decision making using coordination graphs," in International Conference on Advanced Robotics (ICAR), vol. 3, 2003, pp. 1124-1129.

[53] J. Bezanson, A. Edelman, S. Karpinski, and V. B. Shah, "Julia: A fresh approach to numerical computing," SIAM Review, vol. 59, no. 1, pp. 65-98, 2017.

[54] S. Choudhury, K. Solovey, M. J. Kochenderfer, and M. Pavone, "Efficient large-scale multi-drone delivery using transit networks," in IEEE International Conference on Robotics and Automation (ICRA), 2020.

[55] M. Barer, G. Sharon, R. Stern, and A. Felner, "Suboptimal variants of the conflict-based search algorithm for the multi-agent pathfinding problem," in European Conference on Artificial Intelligence (ECAI), 2014, pp. 961-962.

[56] E. Boyarski, A. Felner, R. Stern, G. Sharon, D. Tolpin, O. Betzalel, and S. E. Shimony, "ICBS: improved conflictbased search algorithm for multi-agent pathfinding," in International Joint Conference on Artificial Intelligence (IJCAI), 2015, pp. 740-746. 